\title{
Boundary Value Problems at Infinite Genus
}

\author{
Simon Davis ${ }^{1}$ \\ ${ }^{1}$ Research Foundation of Southern California, Villa La Jolla Drive \#13595, La Jolla, CA, 92039, USA \\ Correspondence: Simon Davis, Research Foundation of Southern California, Villa La Jolla Drive \#13595, La Jolla, CA, \\ 92039, USA. E-mail: sbdavis@ resfdnsca.org
}

\author{
Received: January 14, 2017 Accepted: February 21, 2017 Online Published: March 26, 2017 \\ doi:10.5539/jmr.v9n2p146 URL: https://doi.org/10.5539/jmr.v9n2p146
}

\begin{abstract}
Boundary value problems are formulated on infinite-genus surfaces. These are solved for a variety of boundary conditions. The symbol calculus for differential operators is developed further for solution of parabolic differential equations at infinite genus.
\end{abstract}

\section{MSC: 30E25, 30F35, 34B09, 35K51}

Keywords: parabolic boundary problem, infinite genus surfaces, classical symbols

\section{Introduction}

The theory of partial differential equations with given boundary conditions has developed from series solutions and integral transforms to an operator calculus (Hormander, 1985). The matrix algebra formed from the symbols representing a set of operators for a pseudodifferential equation defined at the interior and the boundary can be used to evaluate the inverse for elliptic boundary value problems (Boutet de Monvel, 1971). A framework for this derivation may be given with this symbolic calculus for parabolic boundary value problems. The Volterra pseudodifferential differential equations were solved for a given set of boundary conditions (Piriou, 1970). It had been proven that an isomorphism existed between a normed space of solutions to elliptic boundary value problems with these boundary conditions in the complex plane on the real line to a normed space of solutions to a corresponding parabolic differential equation (Agronovich and Vishik, 1964). The exponential long-time asymptotics on a noncompact manifold in the elliptic problem (Schuss, 1973) similarly may be transformed to asymptotics in a parabolic problem.

The existence and uniqueness of solutions to parabolic differential equations with exponential asymptotics in the $t \rightarrow \infty$ limit have been established (Krainer, 2002). External states in string amplitudes are known to be described by semiinfinite cylinders and the solutions to equations on these ends in the Euclidean formalism may be related to the parabolic boundary value problems through a generalized inverse Laplace transform. Therefore, the asymptotics of solutions in string theory would be represented. The $t \rightarrow \infty$ limit, however, does not necessarily arise for infinite-genus surfaces with accumulating handles. Exponentially decaying solutions at $t=-\infty$ are not required for the semi-infinite cylinders representing the propagation of external states. Nevertheless, a change of coordinates equivalent to $r=e^{-t}$ again maps the boundary conditions at $t=\infty$ to the origin of a punctured disk at $r=0$.

The methods for solving differential equations of field theories at the ideal boundaries of surfaces will be given. Ideal boundaries may consist of discrete sets of points or a continuum. An example of a series solution to an elliptic differential equation on a surface with a boundary with an infinite number of ends is provided in $\$ 2$. The uniqueness of the function which represents the harmonic measure with a given set of boundary values follows. The path to ideal boundary will be parameterized by a coordinate $t$ tending to infinity, and the mapping from infinity to the origin can have an image that is a discrete set or the real line. When it is not the real line, by the uniformization of surfaces of genus $g \geq 2$, there is a formalism based on the automorphic functions defined on the entire upper half plane instead of a fundamental region for a Fuchsian group, and boundary conditions may be specified on the real line. Infinite-genus surfaces in the class $O_{G}$ are parabolic and have a countable number of ends. The mapping to a surface with one end to a finite region would yield a surface with handles accumulating to only one point. Therefore, the boundary is not identified with a continuum. The boundary value problem shall be solved through the above method of functions invariant under the uniformizing group.

Analytic function theory on infinite-genus surfaces is necessary for the set of conditions (Widom,1971) required for a convergent representation of the Green function as a product over elements of the uniformizing group (Pommerenke, 1976) The convergence of the series for theta functions similarly can be proven for spectral curves of the parabolic heat equation. Nontrivial solutions $\psi \in L_{l o c}^{\infty}\left(\mathbb{R}^{2}\right)$ to the heat equation $\left(\frac{\partial}{\partial t}-\frac{\partial^{2}}{\partial x_{2}^{2}}\right) \psi+q\left(x_{1}, x_{2}\right) \psi=0$ and $\psi\left(x_{1}+\omega_{1}, x_{2}+\omega_{2}\right)=\xi_{1} \psi\left(x_{1}, x_{2}\right)$ and $\psi\left(x_{1}, x_{2}+2 \pi\right)=\xi_{2} \psi\left(x_{1}, x_{2}\right)$ for $q \in L^{2}\left(\mathbb{R}^{2} / \Gamma\right)$, where $\Gamma=(0,2 \pi) \mathbb{Z} \oplus\left(\omega_{1}, \omega_{2}\right) \mathbb{Z}$, may be mapped to functions that satisfy 
the Kadomtsev-Petviashvili equation on $\mathbb{R}^{2} / \Gamma$ (Feldman, Knorrer Trubowitz, 2003). The pseudodifferential calculus for this parabolic boundary value problem in $\$ 3$ then yields a solution for the Kadomtsev-Petviahshvili equation in terms of this theta function through the mapping to the hyperbolic space the covering transformation of the surface.

\section{The Harmonic Measure of the Ideal Boundary}

The Dirichlet problem may be formulated for an infinite-genus surface. If $\Delta u=0$ with specified values at the boundary, $w(z, \partial \Sigma, \Sigma)$, which equals the Perron solution at $z$, gives the probability of random motion beginning at $z$ and exiting through $\partial \Sigma$ (Kakutani, 1944). It equals the harmonic measure of the ideal boundary. Random motion does not produce a flux to the embedding space unless the the capacity is non-zero, and the surface does not belong to $O_{G}$. This result would complement the introduction of exceptional group gauge symmetries through the intersection matrix (Davis, 2014).

The capacity of the ideal boundary is $c_{\beta}=e^{-k_{\beta}}$, where

$$
k_{\beta}=\lim _{n \rightarrow \infty} \int_{\partial E_{n}} s_{n} * d s_{n}
$$

with $s_{n}=\ln |z|+\varphi_{n}(z)$ relative to some origin $z=0$ and $\varphi_{n}(z)$ being harmonic on $E_{n}$, an $n^{\text {th }}$ order approximation of the end $E$ (Sario Nakai, 1970). When there is a null boundary, there is no second source for the Green function and $\varphi_{n}(z)$ will not cancel $\ln |z|$ in the limit $z \rightarrow \partial E_{n}$ and $n \rightarrow \infty$. If the ideal boundary has non-zero harmonic measure, the equivalent of the second source is sufficient for a cancellation with $\ln |z|$ and the remainder is finite. The integral $\frac{1}{2 \pi} \int_{\beta} s_{\beta} * d s_{\beta}$ would be finite and $c_{\beta} \neq 0$.

The harmonic measure of an end with respect to the ideal boundary is defined to be a solution to $\Delta w=0$ with $\left.w\right|_{\alpha}=0$ and $\left.w\right|_{\beta}=1$. Uniqueness of the harmonic measure follows if it is an $\widetilde{H D}$-minimal function, since another function may be selected to be either less than or greater than $u$ in an entire neighbourhood of the ideal boundary. Any harmonic function with finite Dirichlet norm in $\widetilde{H D}(\Sigma)$ may be expressed as $\int_{D} P(z, p) f(p) d \mu(p)$, where $P(z, p)$ is the harmonic kernel, $f(p)$ is a boundary function and $\mu(p)$ is the harmonic measure (Sario Nakai, 1970).

An example of a surface in $O_{H D}-O_{G}$ is Toki's surface. If $D_{0}$ is the slit disk $D-\cup_{m, n, v} S_{m, n}^{\nu}$, where $S_{m n}^{\nu}=\left\{z=r e^{i \theta} \mid-2^{-2 \mu} \leq\right.$ $\left.\log r \leq-2^{-2 \mu+1}, \theta=v \cdot 2 \pi \cdot 2^{-2 \mu}, v=1, \ldots, 2^{2 \mu}, \mu=2^{m-1}(2 n-1)\right\}$, the Riemann surface is constructed by joining the copies of the disks $\Sigma(i+m j)$ with $\Sigma^{\prime}(i+m+m j)$ for even $j$ and $\Sigma(i+m j)$ with $\Sigma^{\prime}(i-m+m j)$ for odd $j$ cross along every slit $S_{m n}^{v}, n=1,2, \ldots, v=1, \ldots, 2^{2 \mu}$ (Toki, 1962). The Dirichlet problem may be solved on this surface by the integral

$$
\varphi(z)=-\int_{\partial \Sigma} d \sigma \varphi_{0}(\sigma) \frac{\partial G}{\partial n}
$$

where $\left.\varphi\right|_{\partial \Sigma}=\varphi_{0}$ and $G\left(z, z^{\prime}\right)$ is the Green function (Poincare, 1890). The solution on Tôki's surface for the harmonic measure would satisfy the boundary condition $\phi_{0}(\sigma)=1$ at each of the slits in $S_{m n}^{\nu}$.

Theorem 1. The harmonic measure of Tôki's surface may be given in series form.

\section{Proof.}

Given the Green function on the upper half plane $G\left(z, z^{\prime}\right)=-\frac{1}{4 \pi} \ln \frac{\left(x-x^{\prime}\right)^{2}+\left(y-y^{\prime}\right)^{2}}{\left(x-x^{\prime}\right)^{2}+\left(y+y^{\prime}\right)^{2}}$, the normal derivative may be found on the unit disk. Let us define a group $\Gamma_{T}$ generated by translations $\theta_{v} \rightarrow \theta_{v+1}, v=1, \ldots, 2^{2 v}-1, \theta_{2^{2 \mu}} \rightarrow \theta_{1}$ and $m \rightarrow m+1$, $n \rightarrow n+1$. The Green function then can be evaluated by the method of images

$$
G\left(z, z^{\prime}\right)=-\frac{1}{4 \pi} \sum_{\gamma \in \Gamma_{T}} \ln \frac{\left(x-\gamma x^{\prime}\right)^{2}+\left(y-\gamma y^{\prime}\right)^{2}}{\left(x-\gamma x^{\prime}\right)^{2}+\left(y+\gamma y^{\prime}\right)^{2}},
$$

where the slit, $-\frac{1}{4} \leq \log r \leq \frac{1}{8}, \theta=\frac{\pi}{4}$ is aligned with the $y$ axis. At each slit, the normal vector is perpendicular to the direction given by $\gamma\left(S^{1}{ }_{11}\right)$ or $S_{m n}^{v}$. It is sufficient to establish the angle of the perpendicular relative to the adjusted $y$-axis, which is $\frac{\pi}{2}+\theta_{m n}^{v}-\frac{\pi}{4}=\frac{\pi}{4}+\nu(2 \pi) 2^{-2^{m}(2 n-1)}$. The effect of $\gamma_{v}^{s_{1}} \gamma_{m}^{s_{2}} \gamma_{n}^{s_{3}}$ on this angle is

$$
\begin{aligned}
\gamma_{v}^{s_{1}} \gamma_{m}^{s_{2}} \gamma_{n}^{s_{3}}\left(\theta_{m n}^{v}\right) & \left.=\theta^{\left(v+s_{1}\right)_{\sigma\left(2^{2}\right.}\left(m+s_{2}\right)\left(2\left(n+s_{3}\right)-1\right)}\right) \\
& =\frac{\pi}{4}+\left(v+s_{1}\right)_{\sigma\left(2^{\left(m+s_{2}\right)\left(2\left(n+s_{3}\right)-1\right)}\right)}(2 \pi) 2^{-2^{\left(m+s_{2}\right)\left(2\left(n+s_{3}\right)-1\right)}}
\end{aligned}
$$

where $\sigma(N)$ represents cyclic permutation with respect to $N$. The gradient vector perpendular to $\gamma_{v}^{s_{1}} \gamma_{m}^{S_{2}} \gamma_{n}^{s_{3}}\left(S^{\nu}{ }_{m n}\right)$ is 


$$
\begin{aligned}
\cos \left(\frac{\pi}{4}+\right. & \left.\left(v+s_{1}\right)_{\sigma\left(2^{2^{\left(m+s_{2}\right)\left(2\left(n+s_{3}\right)-1\right)}}\right)}(2 \pi) 2^{-2^{\left(m+s_{2}\right)\left(2\left(n+s_{3}\right)-1\right)}}\right) \frac{\partial}{\partial x} \\
& +\sin \left(\frac{\pi}{4}+\left(v+s_{1}\right)_{\sigma\left(2^{\left.2^{\left(m+s_{2}\right)}\right)\left(2\left(n+s_{3}\right)-1\right)}\right)}(2 \pi) 2^{-2^{\left(m+s_{2}\right)\left(2\left(n+s_{3}\right)-1\right)}}\right) \frac{\partial}{\partial y}
\end{aligned}
$$

Consequently,

$$
\begin{aligned}
& \frac{\partial G}{\partial n}=-\frac{1}{4 \pi} \sum_{m=1}^{\infty} \sum_{n=1}^{\infty} \sum_{v=1}^{2^{m}(2 n-1)} \\
& \left\{\cos \left(\frac{\pi}{4}+\left(v+s_{1}\right)_{\sigma\left(2^{2\left(m+s_{2}\right)\left(2\left(n+s_{3}\right)-1\right)}\right)}(2 \pi) 2^{-2^{\left(m+s_{2}\right)\left(2\left(n+s_{3}\right)-1\right)}}\right) \frac{\partial}{\partial x}\right. \\
& \left.+\sin \left(\frac{\pi}{4}+\left(v+s_{1}\right)_{\sigma\left(2^{2\left(m+s_{2}\right)\left(2\left(n+s_{3}\right)-1\right)}\right)}(2 \pi) 2^{-2^{\left(m+s_{2}\right)\left(2\left(n+s_{3}\right)-1\right)}}\right) \frac{\partial}{\partial y}\right\} \\
& \ln \frac{\left(x-\gamma_{v}^{s_{1}} \gamma_{m}^{s_{2}} \gamma_{n}^{s_{3}} x^{\prime}\right)^{2}+\left(y-\gamma_{v}^{s_{1}} \gamma_{m}^{s_{2}} \gamma_{n}^{s_{3}} y^{\prime}\right)^{2}}{\left(x-\gamma_{v}^{s_{2}} \gamma_{m}^{s_{3}} x^{\prime}\right)^{2}+\left(y+\gamma_{v}^{S_{1}} \gamma_{m}^{s_{2}} \gamma_{n}^{s_{3}} y^{\prime}\right)^{2}} .
\end{aligned}
$$

where the action of $\gamma_{v}^{s_{1}} \gamma_{m}^{s_{2}} \gamma_{n}^{s_{3}}$ on points in the disk may be defined by the rotation and radial translation required from the mapping of the midpoint of slit $S^{1}{ }_{11}$ to the midpoint of $S^{1+s_{1}}{ }_{1+s_{2}}, 1+s_{3}$. Then the formula (2) with the Dirichlet boundary condition yields

$$
\begin{aligned}
& \phi(z)=\frac{1}{4 \pi} \int_{\left(x^{\prime}, y^{\prime}\right) \in S^{1}} \sum_{11}^{\infty} \sum_{m=1}^{\infty} \sum_{n=1}^{2^{m}(2 n-1)} \\
& \left\{\cos \left(\frac{\pi}{4}+\left(v+s_{1}\right)_{\sigma\left(2^{2\left(m+s_{2}\right)\left(2\left(n+s_{3}\right)-1\right)}\right)}(2 \pi) 2^{-2^{\left(m+s_{2}\right)\left(2\left(n+s_{3}\right)-1\right)}}\right) \frac{\partial}{\partial x}\right.
\end{aligned}
$$

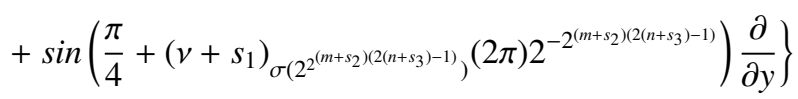

$$
\begin{aligned}
& \ln \frac{\left(x-\gamma_{v}^{S_{1}} \gamma_{m}^{S_{2}} \gamma_{n}^{S_{3}} x^{\prime}\right)^{2}+\left(y-\gamma_{v}^{S_{1}} \gamma_{m}^{S_{2}} \gamma_{n}^{S_{3}} y^{\prime}\right)^{2}}{\left(x-\gamma_{n}^{S_{2}} \gamma_{n}^{S_{3}} x^{\prime}\right)^{2}+\left(y+\gamma_{v}^{S_{1}} \gamma_{m}^{S_{2}} \gamma_{n}^{S_{3}} y^{\prime}\right)^{2}} .
\end{aligned}
$$

The uniqueness of this solution follows from evaluating the difference of two harmonic measures with same boundary conditions to be a harmonic function vanishing everywhere on the boundary. By the maximum modulus principle, this harmonic function vanishes and the two series are equal.

\section{Solutions Spaces for Parabolic Surfaces of Infinite Genus}

The identification of theta function on the Riemann surface of infinite genus and periodic solutions to the KadomtsevPetviashvilli equation on $\mathbb{R}^{2}$ reflects an embedding into a calculus of symbols for this parabolic differential equation that would unify the two analytic function spaces.

The solutions to differential equations on surfaces of infinite genus belong to Sobolev spaces $W_{s}^{p}$. Given that the Hilbert spaces $\mathcal{H}$ and $\tilde{\mathcal{H}}$ admit a scaling group action, $\Lambda$ is a conical manifold and the norm is $\langle\xi, \lambda\rangle_{\ell}=\left(1+|\xi|^{2 \ell}+\|^{2}\right)^{\frac{1}{2 \ell}}$, which satisfies a linear convexity relation, the space of $\mathcal{L}(\mathcal{H}, \tilde{\mathcal{H}})$ anisotropic symbols of order $\mu, \Lambda$ (Krainer, 2002) is

$S^{\mu ; \ell}\left(\mathbb{R}^{n} \times \Lambda ; \mathcal{H}, \tilde{\mathcal{H}}\right)=\left\{a \in C^{\infty}\left(\mathbb{R}^{n} \times \Lambda, \mathcal{L}(\mathcal{H}, \tilde{\mathcal{H}}) ; \forall k \in \mathbb{N}_{0}:\right.\right.$

$\left.\sup _{(x, \lambda) \in \mathbb{R}^{n} \times \Lambda}\left\|\tilde{\kappa}_{\langle\xi, \lambda\rangle_{\ell} \leq k}^{-1} \partial_{(\xi, \lambda)}^{\beta} a(\xi, \lambda) \kappa_{\langle\xi, \lambda\rangle_{\ell}}\right\|\langle\xi, \lambda\rangle_{\ell}^{-\mu+|\beta|_{\ell}}<\infty\right\}$ where $\kappa_{\rho} \in \mathcal{L}(\mathcal{H})$ and $\tilde{\kappa}_{\rho} \in \mathcal{L}(\tilde{\mathcal{H}})$ with $\rho \in \mathbb{R}^{+}$, while the space of classical symbols is $S_{c l}^{\mu ; \ell}\left(\mathbb{H}^{2} \times \Lambda ; \mathcal{H}, \tilde{\mathcal{H}}\right)=\left\{a \in S^{\mu ; \ell}\left(\mathbb{H}^{2} \times \Lambda, \mathcal{H}, \tilde{\mathcal{H}}\right) ; a \sim \sum_{k=0}^{\infty} \chi a_{(\mu-k)}\right\}$ and $\chi \in C^{\infty}\left(\mathbb{H}^{2} \times\right.$ $\Lambda)=\left\{\begin{array}{cc}0 & (x, \lambda)=(0,0) \\ 1 & x \rightarrow \infty\end{array}\right.$ and $a_{(\mu-k)} \in C^{\infty}\left(\mathbb{H}^{2} \times \Lambda \backslash\{(0,0)\}, \mathcal{L}(\mathcal{H}, \tilde{\mathcal{H}})\right.$ consist of anisotropic functions of degree $\mu-k$, with $f\left(\rho \xi, \rho^{\ell} \lambda\right)=\rho^{\mu} \tilde{\kappa}_{\rho} f(\xi, \lambda) \kappa_{\rho}^{-1}$ (Krainer, 2002).

The norm may be defined on the product of the fundamental domain of the uniformizing Fuchsian group of the surface with $\Lambda, \mathcal{F}_{\Gamma} \times \Lambda=\mathbb{H}^{2} / \Gamma \times \Lambda$ to be

$$
\langle\xi, \lambda\rangle_{\Sigma, \ell}=\left(1+|\xi|_{\mathbb{H}}^{2 \ell}+|\lambda|^{2}\right)^{\frac{1}{2 \ell}} .
$$

The metric in $\mathbb{H}^{2}$ is $\frac{d x^{2}+d y^{2}}{|y|^{2}}$ and has isometries given by the fractional linear transformations $z \rightarrow \frac{a z+b}{c z+d}$ and fractional antilinear transformations $z \rightarrow \frac{c \bar{z}+d}{a \bar{z}+b}$ with $a d-b c=1$. The composition of two pure translations $z \rightarrow z+b_{1}$ and 
$z \rightarrow z+b_{2}$ yields the pure translation $z \rightarrow z+b_{1}+b_{2}$ with $b_{1}, b_{2} \in \mathbb{R}$. The product of the two groups is isomorphic to $\operatorname{PS} L(2 ; \mathbb{R}) \times P S L(2 ; \mathbb{R})$. It may be noted that fractional linear transformations with complex coefficients are isometries of $\mathbb{H}^{3}$. Since hyperbolic Riemann surfaces would represent boundary components of the space $\mathbb{H}^{3} / G$, where $G$ is a discrete subgroup of $\operatorname{PS} L(2 ; \mathbb{C})$, the restriction to the boundary yields a complex isometry on the covering space $\mathbb{H}^{2}$ if it preserves the upper half plane. The set of translations $z \rightarrow z+b$ where $\operatorname{Im} b>0$ have an image in $\mathbb{H}^{2}$ the composition defines a group. The composition of two translations along geodesics, by contrast, yields a mapping along another geodesic which is not related directly by translation either in $\mathbb{H}^{2}$ or the unit disk with the hyperbolic metric. The first type of translations will be considered in proving the convexity of the anisotropic norm.

Lemma 1. The norm on $\mathbb{H}^{2} / \Gamma$ satisfies the inequality

$$
\left\langle\xi_{1}+\xi_{2}, \lambda_{1}+\lambda_{2}\right\rangle_{\Sigma, \ell}^{|s|} \leq c^{|s|}\left\langle\xi_{1}, \lambda_{1}\right\rangle_{\ell}^{|s|}\left\langle\xi_{2}, \lambda_{2}\right\rangle_{\ell}^{|s|} .
$$

with $c=\max \left(3,1+\frac{3 c_{1}}{\max \left(\left|\xi_{1}\right|_{\mathbb{H}}^{2}\left|\xi_{2}\right|_{\mathbb{H}}^{1}, 2\left|\xi_{1}\right| \mathbb{H}_{\mid}\left|\xi_{2}\right| \mathbb{H}\right)}\right)$ and $\left[\left|\xi_{1}\right|_{\mathbb{H}}^{2 \ell_{1}}\left|\xi_{2}\right|_{\mathbb{H}}^{2 \ell_{2}}\left(\sqrt{2}\left|\xi_{1}\right|_{\mathbb{H}}^{\frac{1}{2}}\left|\xi_{2}\right|_{\mathbb{H}}\right)^{2 \ell_{3}}\right]^{\frac{1}{\ell}} \leq c_{1}$.

\section{Proof.}

The absolute value of $\gamma\left(\xi_{1}+\xi_{2}\right)$ in the hyperbolic plane is

$$
\left|\gamma\left(\xi_{1}+\xi_{2}\right)\right|_{\mathbb{H}}=\frac{\left|\xi_{1}+\xi_{2}\right|_{E}}{\left|\operatorname{Im}\left(\xi_{1}+\xi_{2}\right)\right|_{E}^{2}} \leq \frac{\left|\xi_{1}\right|_{E}}{\left|\operatorname{Im}\left(\xi_{1}\right)\right|^{2}}+\frac{\left|\xi_{2}\right|_{E}}{\left|\operatorname{Im}\left(\xi_{2}\right)\right|^{2}}=\left|\gamma\left(\xi_{1}\right)\right|_{\mathbb{H}}+\left|\gamma\left(\xi_{2}\right)\right|_{\mathbb{H}} .
$$

for any $\gamma \in \Gamma$. It follows that the norm $\langle\xi, \lambda\rangle_{\Sigma, \ell}$ is invariant under the action of $\Gamma$ and can be defined on the fundamental domain. The inequalities

$$
\begin{aligned}
\left\langle\xi_{1}+\xi_{2}, \lambda_{1}+\lambda_{2}\right\rangle_{\ell}^{|s|} & =\left(1+\left|\xi_{1}+\xi_{2}\right|^{2 \ell}+\left|\lambda_{1}+\lambda_{2}\right|^{2}\right)^{\frac{|s|}{2 \ell}} \\
& =\left[1+\left(\frac{\left|\xi_{1}+\xi_{2}\right|_{E}}{\left|\operatorname{Im}\left(\xi_{1}+\xi_{2}\right)\right|^{2}}\right)^{2 \ell}+\left|\lambda_{1}+\lambda_{2}\right|^{2}\right]^{\frac{|s|}{2 \ell}} \\
& \leq\left[1+\left(\frac{\left|\xi_{1}\right|_{E}}{\left|\operatorname{Im}\left(\xi_{1}\right)\right|_{E}^{2}}+\frac{\left|\xi_{2}\right|_{E}}{\left|\operatorname{Im}\left(\xi_{2}\right)\right|_{E}^{2}}\right)^{2 \ell}\right. \\
& \left.+1+\left|\lambda_{1}\right|^{2}+\left|\lambda_{2}\right|^{2}+\left|\lambda_{1} \lambda_{2}\right|^{2}\right]^{\frac{|s|}{2 \ell}} \\
& \leq\left[2+\left(\frac{\left|\xi_{1}\right|_{E}^{2}}{\left|\operatorname{Im}\left(\xi_{1}\right)\right|^{4}}+\frac{\left|\xi_{2}\right|_{E}^{2}}{\left|\operatorname{Im}\left(\xi_{2}\right)\right|^{4}}+2 \frac{\left|\xi_{1} \xi_{2}\right|_{E}}{\left|\operatorname{Im}\left(\xi_{1}\right) \operatorname{Im}\left(\xi_{2}\right)\right|_{E}^{2}}\right)^{\ell}\right. \\
& \leq\left[2+\left.|| \xi_{1}\right|^{2}+\left|\lambda_{2}\right|^{2}+\left|\lambda_{1} \lambda_{2}\right|^{2}\right]^{\frac{|s|}{\ell}} \\
& \left.\left.+\left|\xi_{2}\right|_{\mathbb{H}}^{2}+2\left|\xi_{1}\right||\mathbb{H}| \xi_{2}||_{\mathbb{H}}\right)^{\ell}+\left|\lambda_{2}\right|^{2}+\left|\lambda_{1} \lambda_{2}\right|\right]^{\frac{|s|}{2 \ell}}
\end{aligned}
$$

and

$$
\begin{aligned}
\left|\xi_{1}+\xi_{2}\right|_{\mathbb{H}}^{2 \ell} & \leq\left[\left|\xi_{1}\right|_{\mathbb{H}}^{2}+\left|\xi_{2}\right|_{\mathbb{H}}^{2}+2\left|\xi_{1}\right|_{\mathbb{H}}\left|\xi_{2}\right|_{\mathbb{H}}\right]^{\ell} \\
& \left(1+\frac{3 c_{1}}{\left|\xi_{1}\right|_{\mathbb{H}}^{2}+\left|\xi_{2}\right|_{\mathbb{H}}^{2}+2\left|\xi_{1}\right|_{\mathbb{H}}\left|\xi_{2}\right|_{\mathbb{H}}}\right)^{\ell},
\end{aligned}
$$

where

$$
\left|\xi_{1}\right|_{\mathbb{H}}^{2 \ell_{1}}\left|\xi_{2}\right|^{2 \ell_{2}}\left(\left|\xi_{1}\right| \mathbb{H}\left|\xi_{2}\right| \mathbb{H}\right)^{2 \ell_{3}} \leq c_{1}^{\ell}
$$

yield 


$$
\begin{aligned}
\left\langle\xi_{1}+\xi_{2}, \lambda_{1}+\lambda_{2}\right\rangle_{\ell}^{|s|} \leq & c^{|s|}\left[3+\left|\lambda_{1}\right|^{2}+\left|\lambda_{2}\right|^{2}+\left|\lambda_{1} \lambda_{2}\right|^{2}+\left|\xi_{1}\right|_{\mathbb{H}}^{2 \ell}+\left|\xi_{2}\right|_{\mathbb{H}}^{2 \ell}\right. \\
& \left.+\left(\left|\xi_{1}\right| \mathbb{H} \xi_{2} \mid \mathbb{H}\right)^{2 \ell}+\left|\lambda_{1}\right|^{2}\left|\xi_{2}\right|_{\mathbb{H}}^{2 \ell}+\left|\lambda_{2}\right|^{2}\left|\xi_{1}\right|_{\mathbb{H}}^{2 \ell}\right]^{\frac{|s|}{2 \ell}} \\
\leq & c^{|s|}\left\langle\xi_{1}, \lambda_{1}\right\rangle_{\ell}^{|s|}\left\langle\xi_{2}, \lambda_{2}\right\rangle_{\ell}^{|s|}
\end{aligned}
$$

with $c=\max \left(3,1+\frac{3 c_{1}}{\max \left(\left|\xi_{1}\right|_{\mathbb{H}}^{2},\left|\xi_{2}\right|_{\mathbb{H}}^{2}, 2\left|\xi_{1}\right|_{\mathbb{H}}\left|\xi_{2}\right|_{\mathbb{H}}\right)}\right)$.

The space of Volterra symbols is defined by the space of classical symbols with the parameter space $\Lambda$ equal to $\mathbb{H}$. The Volterra symbols are given by

$$
\begin{aligned}
& a(\xi, \zeta)=\sum_{k=1}^{\infty} \chi\left(\frac{\xi}{c_{k}}, \frac{\zeta}{c_{k}^{k}}\right) a_{k}(\xi, \zeta)=\sum_{k=1}^{\infty}\left(H\left(\varphi\left(c_{k} t\right) a_{k}\right)(\xi, \zeta), c_{k} \rightarrow \infty \text { as } k \rightarrow \infty,\right. \text { and } \\
&(H(\varphi) b)(\xi, \zeta)=\int_{\mathbb{H}^{2}} e^{-i t \tau} \varphi(t) b(\xi, \eta-\tau) d t d \tau{ }_{V}^{\sim} \sum_{j=0}^{\infty}\left(\frac{-1)^{j}}{j !} D_{t}^{j} \varphi(0)\right) \partial_{\zeta}^{j} b(\xi, \zeta)
\end{aligned}
$$

and the translation operator is $\left(T_{i \tau} a\right)(\xi, \zeta)=a(\xi, \zeta+i \tau)$ (Krainer, 2002). The operator product for the symbols would modified to

$$
a \# b(x, \xi, \zeta)=\iint e^{-i y \eta} a(x, \xi+\eta, \zeta) b(x+y, \xi, \zeta) \frac{d y}{|\operatorname{Im}(y)|^{2}} d \eta_{V}^{\sim} \sum_{\alpha \in \mathbb{N}_{0}^{n}} \frac{1}{\alpha !}\left(\partial^{\alpha} a\right)\left(D_{x} b\right) .
$$

The operator algebra is defined such that each symbol $a$ has an inverse $p$ with $a \# p-1$ and $p \# a-1$ is a symbol of order $-\infty$ belonging to $S^{-\infty: \ell}\left(\mathbb{H}^{2} \times \Lambda, H, \tilde{H}\right)=\left\{a \in C^{\infty}\left(\mathbb{H}^{2} \times \Lambda, \mathcal{L}(H, \tilde{H})\right) ; \forall k \in \mathbb{N}_{0}: \sup _{\substack{(\xi, \lambda) \in \mathbb{H}^{2} \times \Lambda \\ \mid \beta_{\ell}<k}}\left\|\tilde{\kappa}_{\langle\xi, \lambda\rangle_{\ell}}^{-1} \partial_{(\xi, \lambda)}^{\beta} a(\xi, \lambda) \kappa_{\langle\xi, \lambda\rangle \ell}\right\|\right.$

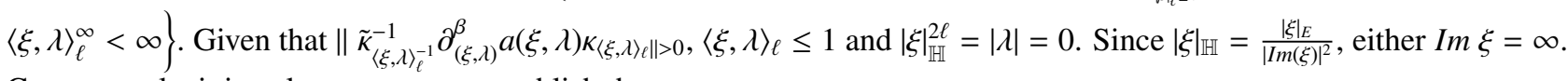
Consequently, it is only necessary to establish that

$$
\begin{aligned}
\left\|\tilde{\kappa}_{\langle\sigma \pm i \infty, 0\rangle}^{-1} \partial_{(\sigma+i \infty, 0)}^{\beta} a(\sigma+i \infty, 0) \kappa_{\langle\sigma+i \infty, 0\rangle}\right\| & =\left\|\tilde{\kappa}_{1}^{-1} \partial_{\sigma}^{\beta} a(\sigma+i \infty, 0) \kappa_{1}\right\| \\
& =\left\|\partial_{\sigma}^{\beta} a(\sigma+i \infty, 0)\right\|<\infty .
\end{aligned}
$$

since $1+|\beta|^{2} \leq k$ includes $\beta=0$. Then $a(\xi, 0)$ must be bounded at infinity in all directions in the upper half plane. Furthermore, if $\xi \in \mathbb{F}_{\Gamma}$ has finite coordinates, $\langle\xi, \lambda\rangle_{\ell}>1$ and

$$
\left\|\tilde{\kappa}_{\langle\xi, \lambda\rangle_{\ell}}^{-1} \partial_{(\xi, \lambda)}^{\beta} a(\xi, \lambda) \kappa_{\langle\xi, \lambda\rangle}\right\|=0
$$

and $a(\xi, \lambda)$ must vanish in this space.

The operator symbol with respect to a boundary parameterized by $x$ is

$$
\begin{gathered}
o p_{x}(a): S_{c l}^{\mu ; \ell}\left(\mathbb{H}^{2} \times \mathbb{H}^{2} \times \Lambda ; \mathbb{C}^{N_{-}}, \mathbb{C}^{-}\right) \rightarrow S^{\mu ; \ell}\left(\mathbb{R} \times \mathbb{R} \times \Lambda ; H^{s, \delta}\left(\mathbb{R}_{+}, \mathbb{C}^{N_{-}}\right), H^{s-\mu, \delta}\left(\mathbb{C}^{N_{-}}\right)\right) \\
s>-\frac{1}{2}, \delta \in \mathbb{R} .
\end{gathered}
$$

Near $r=0$, the smoothing Mellin operator is

$$
o p_{M}^{\gamma-1}(h) u(r)=\frac{1}{2 \pi i} \int_{\Gamma_{\frac{3}{2}-\gamma}} \int_{\mathbb{R}_{+}}\left(\frac{r}{r^{\prime}}\right)^{-s} h(r, z) u\left(r^{\prime}\right) \frac{d r^{\prime}}{r^{\prime}} d z
$$

The calculus of classical symbols includes singular Green, trace, potential and boundary symbols (Schrohe, 2001). The operator for the Green function can be expanded as $g=\sum_{j=0}^{d} g_{j} \partial_{x}^{j} \in S_{c l}^{\mu ; \ell}\left(\mathbb{R} \times \mathbb{R} \times \Lambda, S^{\prime}\left(\mathbb{R}_{+}\right), S\left(\mathbb{R}^{+}\right) \otimes \mathcal{L}\left(\mathbb{C}^{N_{-}}, \mathbb{C}^{N_{+}}\right)\right)$, with $\mathcal{S}\left(\mathbb{R}_{+}\right) \simeq \operatorname{proj}-\lim _{s, \delta \in \mathbb{R}} H^{s, \delta}\left(\mathbb{R}_{+}\right)$and $S^{\prime}\left(\mathbb{R}_{+}\right) \simeq \operatorname{ind}-\lim _{s, \delta \in \mathbb{R}} H_{0}^{s, \delta}\left(\overline{\mathbb{R}}_{+}\right)$and $g_{j}$ is a symbol of order $\mu-j$ and type 0 , the trace symbol of order $\mu$ and type $d$ equals $\left.t=\sum_{j=0}^{d} t_{j} \partial_{x}^{j} \in S_{c l}^{\mu ; \ell}\left(\mathbb{R} \times \mathbb{R} \times \Lambda, \mathcal{S}^{\prime}\left(\mathbb{R}_{+}\right), \mathbb{C}\right) \otimes \mathcal{L}\left(\mathbb{C}^{N_{-}}, \mathbb{C}^{M_{+}}\right)\right)$, where $t_{j}$ is a symbol of order $\mu-j$ and type 0 , the potential symbol of order $\mu, k \in S_{c l}^{\mu ; \ell}\left(\mathbb{R} \times \mathbb{R} ; \mathbb{C}, S\left(\mathbb{R}_{+}\right) \otimes \mathcal{L}\left(\mathbb{C}^{M_{-}}, \mathbb{C}^{N_{+}}\right)\right.$, with $N_{-}, N_{+}$and $M_{-}, M_{+}$being the complex dimension of the domain and range of symbols satisfies the transmission condition (Boutet 
de Monvel, 1971), requiring an upper bound for derivatives with homogeneous components, and the more general class, respectively, and the boundary symbol of order $\mu$ and type $d$ is given by

$$
a_{0}=\left(\begin{array}{cc}
o p_{x}(a)+g & k \\
t & s
\end{array}\right)
$$

with the classical symbols being defined to be automorphic with respect to $\Gamma, a(\Gamma \xi, \lambda)=a(\xi, \lambda)$ (Krainer, 2002). It can be proven that $g=\sum_{j=0}^{d-1} k_{j} \gamma_{j}+g_{0}$, where $k_{j}$ is a potential symbol of order $\mu-j-\frac{1}{2}, \gamma_{v}(f)=\partial_{x}^{v} f$ is a trace symbol of order $v+\frac{1}{2}$,

$$
a_{0} \# b_{0}=\left(\begin{array}{cc}
o p_{x}\left(a \# \#_{x} b\right)+\tilde{g} & \tilde{k} \\
\tilde{t} & \tilde{s}
\end{array}\right)
$$

a boundary symbol of order $\mu_{1}+\mu_{2}$ and type $d=\max \left(\mu_{2}+d_{1}, d_{2}\right)$ with $a_{0}=\left(\begin{array}{cc}o p_{x}(a)+g_{1} & k_{1} \\ t_{1} & s_{1}\end{array}\right)$ and $b_{0}=\left(\begin{array}{cc}o p_{x}(b)+g_{2} & k_{2} \\ t_{2} & s_{2}\end{array}\right)$ are boundary symbols of order and type $\left(\mu_{1}, d_{1}\right)$ and $\left(\mu_{2}, d_{2}\right)$ respectively.

Boundary value problems on a manifold $X$ of dimension $\mathrm{n}$ would be formulated on a boundary $Y$ of dimension $n-1$. The space of classical symbols have been defined with $X$ and $Y$ chosen to be $\mathbb{H}^{2}$ and $\mathbb{R}$ respectively. The ideal boundary of a Riemann surface is given by $(\mathcal{F} \cap \mathbb{R}) / \Gamma$. A differential operator $A=\sum_{j=0}^{M} A_{j}(t) \partial_{t}^{j}=\sum_{j=0}^{M} A_{j}(-\ln r)\left(-r \partial_{r}\right)^{j}$ and the solutions to $A u=f$ has the form $\sum_{j} \sum_{k=0}^{m_{j}} \tilde{c}_{j, k} \log ^{k}(r) r^{-p_{j}}$ as $r \rightarrow 0$. The data at the boundary determine the Mellin asymptotic type $\left\{\left(p_{j}, m_{j}, L_{j}\right), j \in \mathbb{Z}\right\}$, where $m_{j} \in \mathbb{N}_{0}, L_{j}$ are finite-dimensional operators of $\mathcal{B}^{-\infty, d}(X)$ and $p_{j} \in \mathbb{C}$ such that $a(z)=\sum_{k_{j}=0}^{m_{j}} v_{k_{j}}\left(z-p_{j}\right)^{-\left(k_{j}+1\right)}+a_{0}(z)$.

Given a matrix

$$
\left(\begin{array}{ll}
A & K \\
T & Q
\end{array}\right)
$$

and the inverse

$$
\left(\begin{array}{ll}
\hat{P} & \hat{K} \\
\hat{T} & \hat{Q}
\end{array}\right)
$$

provides a solution to the differential equation. Letting $\omega A \tilde{\omega}=o p_{M}^{\gamma-1}(h)+A_{M+G}$ near $r=0, \omega, \tilde{\omega} \in C_{0}^{\infty}\left(\bar{R}_{+}\right), A_{M+G}$ is a Green operator of type $d, \tilde{h}=\left(H_{\gamma-1} h^{\prime}\right)(r, z)$ is the interior symbol, $P^{\prime}=\omega_{1} o p_{M}^{\gamma-1}(\tilde{h}) \omega_{2}+\left(1-\omega_{1}\right) o p_{r}\left(\tilde{a}\left(1-\omega_{3}\right)\right.$, with $\chi_{\left[0, \tilde{T}_{1}\right]}<\omega_{3}<\omega_{1}<\omega_{2}<\chi_{\left.0, \tilde{T}_{1}\right]}, \tilde{P}=P^{\prime}+\omega o p_{M}^{\gamma-1}(g) \omega$, where $g=\sigma_{M}^{0}(A)^{-1}-\sigma_{M}^{0}\left(P^{\prime}\right) \in M_{V, Q}^{-\infty, t^{\prime}}\left(X ; \mathbb{H}_{\frac{3}{2}-\gamma}\right), P=\tilde{P}\left(1+D_{1}\right)$ or $P=\left(1+D_{2}\right) \tilde{P}$ satisfies $P A=1$ (Krainer, 2002). Then $u=P^{-1} f$.

This technique easily transposes between $\mathbb{R}^{2}$ and $\mathbb{H}^{2}$ since the classical symbols had been defined initially for $X=\mathbb{R}^{n}$. The following lemma provides a mapping between solutions of elliptic and parabolic boundary value problems restricted by conditions on the function in the upper half plane.

Lemma 2. The solution to a parabolic boundary value problem with conditions on the real line is given by a generalized Fourier transform of a function satisfying an elliptic differential equation.

\section{Proof.}

Let $u(x, t)$ be a solution to an elliptic equation

$$
\frac{\partial^{2} u}{\partial t^{2}}+k \frac{\partial^{2} u}{\partial x^{2}}=0
$$

Let $U(x, p)$ be defined such that $\frac{\partial U(x, p)}{\partial p}$ is the inverse Laplace transform of $\frac{\partial u(x, t)}{\partial t}$ such that

$$
U(x, p)=\frac{1}{2 \pi i} \int_{0}^{p} d \tilde{p} \int_{\rho-i \infty}^{\rho+i \infty} e^{\tilde{p} t} \frac{\partial u(x, t)}{\partial t} d t .
$$

where $t$ is generalized to be a complex variable and the line $\operatorname{Re} t=\rho$ is located to the right of any singularities of $\frac{\partial u(x, t)}{\partial t}$. Suppose that

$$
\frac{\partial U(p, x)}{\partial p}=\kappa \frac{\partial^{2} U(p, x)}{\partial x^{2}} .
$$

Then

$$
\int_{\rho-i \infty}^{\rho+i \infty} e^{p t} \frac{\partial u(x, t)}{\partial t} d t=\kappa \int_{\rho-i \infty}^{\rho+i \infty} e^{p t} \frac{\partial^{2} u(x, t)}{\partial t^{2}} d t
$$


and

$$
\frac{1}{p} \int_{\rho-i \infty}^{\rho+i \infty} \frac{\partial}{\partial t}\left(e^{p t}\right) \frac{\partial u(x, t)}{\partial t} d t=\kappa \int_{\rho-i \infty}^{\rho+i \infty} e^{p t} \frac{\partial^{2} u(x, t)}{\partial t^{2}} d t .
$$

After integration by parts, setting the boundary terms equal to zero,

$$
-\frac{1}{p} \int_{\rho-i \infty}^{\rho+i \infty} e^{p t} \frac{\partial^{2} u(x, t)}{\partial t^{2}} d t=\kappa \int_{\rho-i \infty}^{\rho+i \infty} e^{p t} \frac{\partial^{2} u(x, t)}{\partial t^{2}} d t .
$$

The equation derived from the integrand is

$$
\frac{1}{p} \frac{\partial^{2} u(x, t)}{\partial t^{2}}+\kappa \frac{\partial^{2} u(x, t)}{\partial x^{2}}=0
$$

which is the elliptic equation with $k=\kappa p$. The space of functions with $\frac{\partial u(x, t)}{\partial t}$ defined in the plane $\operatorname{Re} t>\gamma$ such that $\left\|\frac{\partial u(x, t)}{\partial t}\right\|_{\alpha}^{2}=\left.\sup _{\sigma>\gamma} \int\left|\frac{\partial u(x, t)}{\partial t}\right|_{t=\sigma+i \tau}\right|^{2}|\sigma+i \tau|^{2 \alpha} d \tau<\infty$ is mapped to functions of the form $\frac{\partial U(x, p)}{\partial p}$ defined on the real line and equal to zero for $\operatorname{Re} p<0$, with $e^{-\gamma p} \frac{\partial U(x, p)}{\partial p} \in H_{\alpha}^{1}(\mathbb{R})$ for $\operatorname{Re} \gamma>\rho$ and $\left\|\frac{\partial U(x, p)}{\partial p}\right\|_{\alpha}^{2}=\left(\int_{\mathbb{R}}\left(1+|\xi|^{2 \alpha}\right)\left|\frac{1}{2 \pi} \int_{-\infty}^{\infty} e^{-i \xi p} \frac{\partial U(x, p)}{\partial p}\right|^{2} d \xi\right)^{2}<$ $\infty$ (Agronovich \& Vishik, 1964).

The region of support in the $p$ plane can be rotated by $\frac{\pi}{2}$ to the upper half-plane. It follows that the method may be used to invert the differential operator on $\mathbb{R}^{2} / \Gamma_{1}$ and $\mathbb{H}^{2} / \Gamma_{2}$. The mapping between the solutions with the specified asymptotic data follows from the relation between the integral transformations.

Consequently, it is necessary to consider the eigenvalue spectrum of the heat equation on $\mathbb{R}^{2} / \Gamma$ and the KadomtsevPetviashvilli equation. The mapping between the calculus of symbols for the this equation and the heat curve is given in the following theorem.

Theorem 2. There exists a transformation $\Phi$ from the class symbols of the heat equation operator on a curve of infinite genus and the Kadomtsev-Petviashvili equation on $\mathbb{R}^{2}$ with periodic boundary conditions.

Proof. The Kadomtsev-Petviashvili equation with periodic boundary conditions and the differential equation defining the heat curve may be formulated on $\mathbb{R}^{2} / \Gamma_{1}$ and $\mathbb{H}^{2} / \Gamma_{2}$ respectively for discrete group $\Gamma_{1}$ and $\Gamma_{2}$. The group $\Gamma_{1}$ is the infinite tensor product of $\Gamma_{p e r}$. generated by two lattice vectors $(0,2 \pi)$ and $\left(\omega_{1}, \omega_{2}\right)$ for each admissible value of $\left(\xi_{1}, \xi_{2}\right)$. It is not possible to formulate the solution on $\mathbb{R}^{2} / \Gamma_{\text {per }}$. because iterations of the boundary condition for $\xi_{1} \neq 1$ and $\xi_{2} \neq 1$ yield a different values of $\psi\left(x_{1}, x_{2}\right)$ at each lattice point. Instead, the quotient will be a surface consisting of an infinite sequence of genus-one components with the same monodromy factor for each solution. The Fuchsian group $\Gamma_{2}$ defined by the set of periodicity factors $\left(\xi_{1}, \xi_{2}\right) \in \mathbb{C}^{*} \times \mathbb{C}^{*}$ corresponding to nontrivial $\psi\left(x_{1}, x_{2}\right) \in L^{2}\left(\mathbb{R}^{2} / \Gamma_{1}\right)$ in the heat equation, requires dual group $\Gamma^{\#}=\left(\frac{2 \pi}{\omega_{1}}, 0\right) \mathbb{Z} \oplus\left(-\frac{\omega_{2}}{\omega_{1}}, 1\right) \mathbb{Z}$, the operator $H_{k}=e^{-i\langle k, x\rangle}\left(\frac{\partial}{\partial x_{1}}-\frac{\partial^{2}}{\partial x_{2}^{2}}\right) e^{i\langle k, x\rangle}=\frac{\partial}{\partial x_{1}}-2 i k_{1} \frac{\partial}{\partial x_{2}}-\frac{\partial^{2}}{\partial x_{2}^{2}}+i k_{1}+k_{2}^{2}$ and the union of the parabolas $\mathcal{H}(0)=\cup_{b \in \Gamma^{*}} \mathcal{P}_{b}, \mathcal{P}_{b}=\left\{\left(k_{1}, k_{2}\right) \in \mathbb{C}^{2} \mid \mathcal{P}_{b}\left(k_{1}, k_{2}\right)=i\left(k_{1}+b_{1}\right)+\left(k_{2}+b_{2}\right)^{2}=0\right\}$ (Feldman, Knorrer Trubowitz, 2003). Therefore, condition on the periodicity factors is translated to a condition on eigenvalues of a related differential operator.

Following the integral representation of operator symbols and products on $\mathbb{R}^{2}$ and $\mathbb{H}^{2}$, the mapping $\Phi$ will be defined by

$$
\Phi:\left(\begin{array}{cc}
A & K \\
T & Q
\end{array}\right)_{\mathbb{R}^{2} / \Gamma_{1}} \rightarrow\left(\begin{array}{cc}
A & K \\
T & Q
\end{array}\right)_{\mathbb{H}^{2} / \Gamma_{2}}
$$

such that the transformation of the boundary symbol

$$
\Phi_{B}:\left(\begin{array}{cc}
o p_{x}(a)+g & k \\
t & s
\end{array}\right)_{\mathbb{R}^{2} / \Gamma_{1}} \rightarrow\left(\begin{array}{cc}
o p_{x}(a)+g & k \\
t & s
\end{array}\right)_{\mathbb{H}^{2} / \Gamma_{2}}
$$

is a continuous limit of $\Phi$ at the boundary $\mathbb{R}^{2} / \Gamma_{1} \cap \mathbb{R}$. For a parabolic symbol, the boundary symbol is defined on a set of null harmonic measure. The inverse of the mapping

$$
\Phi^{-1}:\left(\begin{array}{cc}
A & K \\
T & Q
\end{array}\right)_{\mathbb{H}^{2} / \Gamma_{2}}^{-1} \equiv\left(\begin{array}{cc}
\hat{P} & \hat{K} \\
\hat{T} & \hat{Q}
\end{array}\right)_{\mathbb{H}^{2} / \Gamma_{2}} \rightarrow\left(\begin{array}{cc}
A & K \\
T & Q
\end{array}\right)_{\mathbb{R}^{2} / \Gamma_{1}}^{-1} \equiv\left(\begin{array}{cc}
\hat{P} & \hat{K} \\
\hat{T} & \hat{Q}
\end{array}\right)_{\mathbb{R}^{2} / \Gamma_{1}}
$$


with $\hat{P}$ yielding the inverse $P$ of the differential operator $A$. It follows that solutions to the heat equation on the infinitegenus curve $\mathbb{H}^{2} / \Gamma_{2}$, including the theta function, would be mapped to a solution of the Kadomtsev-Petviashvili equation on $\mathbb{R}^{2}$ with periodic boundary conditions. The independence of the eigenvalue spectrum and the heat curve with respect to the time parameter allows an interpretation of the Green function of the differential operator in terms of the eigenvalues through $G\left(x_{1}, x_{2}\right)=\sum \frac{\psi_{n}^{*}\left(x_{1}\right) \psi_{n}\left(x_{2}\right)}{\lambda_{m}-\lambda}$. Consequently, the differential operator also can be represented by the eigenvalue spectrum and the infinite-genus curve. The coordinates on the Riemann surface may be derived from local complex coordinates with an expansion of the holomorphic one-forms in terms of the differentials of these coordinates together with vectors representing multiplicative coefficients (Feldman, Knorrer Trubowitz, 2003). It follows that the map $\Phi$ from the theta function on the Riemann surface to the solution to the Kadomtsev-Petviashvili equation only requires that the surface satisfies standard geometric hypotheses and bounds on the coefficients vectors in the theta function.

The introduction of a map from one differential system to another defined on a different domain may yield a method for solving a similar class of equations.

\section{Conclusion}

There exist infinite-genus surfaces with ideal boundaries that are represented as images under an infinite group of a single component. Then the Dirichlet problem may be solved through the method of images. The Green function can be expressed as an infinite sum of functions on the upper half plane with an infinite number of sources. The solution to the boundary value problem is an integral of the product of the field on a single component and the normal derivative of the Green function.

The formulation of the class of symbols directly on the hyperbolic plane facilitates the study of differential equations on Riemann surfaces of genus $g \geq 2$. The class symbols has been given on a manifold of the form $X \times\left[t_{0}, \infty\right)$, where $X$ is $\mathbb{R}^{n}$. The dimension has been set equal to 2 and the integrals representing the symbols have been generalized to $\mathbb{H}^{2}$. Norm conditions have been demonstrated to be valid in hyperbolic space, which is necessary for the definition of equivalence and symbols with order $-\infty$. Consequently, it is possible to transform symbols and operators from $\mathbb{R}^{2}$ to $\mathbb{H}^{2}$.

Together with the quotient by the discrete uniformizing group, it has been found that there exists a transformation between a theta function on a Riemann surface of infinite genus and the solution to the Kadomtsev-Petviashvili equation satisfying periodic conditions. It is the operator between the two domains. The form of the solution requires the mapping to represent the definition of the holomorphic one-forms and the theta functions in terms of coordinates on the complex plane. This technique may have some degree of generality because an infinite symmetry with respect to one component of the surface is not necessary and mappings to domains with tractable boundary value problems would provide a method for deriving the solution.

\section{References}

Agronovich, M. S., \& Vishik, M. I. (1964). Elliptic Problems with a Parameter and Parabolic Problems of General Type. Russ. Math. Surveys, 19, 53-157. https://doi.org/10.1070/RM1964v019n03ABEH001149

Boutet de Monvel, L. (1971). Boundary Problems for Pseudo-Differential Operators. Acta Math. 126, 11-51. https://doi.org/10.1007/BF02392024

Davis, S. (2014). The Intersection Form of Four-Manifolds and Exceptional Group Symmetries. Research Foundation of Southern California preprint, RFSC-14-40.

Feldman, J., Knörrer, H., \& Trubowitz, E. (2003). Riemann Surfaces of Infinite Genus. CRM Monograph Series, 20. American Mathematical Society, Providence.

Hormander, L. (1985). The Analysis of Linear Partial Differential Operators. Vol. III. Springer-Verlag, Heidelberg.

Kakutani, S. (1944). Two-Dimensional Brownian Motion and Harmonic Functions. Proc. Imp. Acad. Tokyo, 20, 706-714. https://doi.org/10.3792/pia/1195572706

Krainer, T. (2002). Volterra Families of Pseudodifferential Operators, In J. Gil, D. Grieser \& M. Lesch (Eds.), Approaches to Singular Analysis: A Volume of Advances in Partial Differential Equations. Operator Theory: Advances and Applications, 138, 1-45. Berlin: Birkhauser Verlag. https://doi.org/10.1007/978-3-0348-8191-31

Poincare, H. (1890). Sur les Equations aux Dérivées Partielles de la Physique Mathematique. Amer. Math. J., 12, 211-294. https://doi.org/10.2307/2369620

Piriou, A. (1970). Une classe d'operateurs pseudo-differentiels du type de Volterra. Ann. Inst. Fourier Grenoble, 20, 77-94. https://doi.org/10.5802/aif.339 
Pommerenke, Ch. (1976). On the Green's Function of Fuchsian Groups, Ann. Academiae Scientiarum Fennicae, Ser. A. I. Mathematica, 2, 409-427.

Sario, L., \& Nakai, M. (1970). Classification Theory of Riemann Surfaces, Springer-Verlag, Berlin.

Schrohe, E. (2001). A Short Introduction to Boutet de Monvel's Calculus in Approaches to Singular Analysis, In J. Gil, D. Grieser and M. Lesch (Eds.), Advances in Partial Differential Equations. Oper. Theory, Adv. Appl, 125, 85-116. Berlin: Birkhauser Verlag.

Schuss, Z. (1973). Asymptotic Expansions for Parabolic Systems, J. Math. Anal. Appl., 4, 136-159. https://doi.org/10.1016/0022-247X(73)90032-2

Tôki, Y. (1952). On the Classification of Open Riemann Surfaces, Osaka Math. J., 4, 191-201.

Widom, H. (1971). The Maximum Principle for Multiple-Valued Analytic Functions, Acta. Math., 126, 63-82.

\section{Copyrights}

Copyright for this article is retained by the author(s), with first publication rights granted to the journal.

This is an open-access article distributed under the terms and conditions of the Creative Commons Attribution license (http://creativecommons.org/licenses/by/4.0/). 